\title{
ENVISAT ASAR Data Reduction: Impact on SAR Interferometry
}

\author{
Ian H. McLeod, Ian G. Cumming, Member, IEEE, and Michael S. Seymour, Student Member, IEEE
}

\begin{abstract}
An onboard synthetic aperture radar (SAR) signaldata reduction algorithm called Flexible Block Adaptive Quantization (FBAQ) was developed in 1994 for the Advanced SAR (ASAR) on the ENVISAT satellite. This paper presents work done in a follow-on study that examined the impact of the datareduction algorithm on the accuracy of two digital elevation models (DEM) produced by using the technique of repeat-pass SAR interferometry. All three allowable data compression ratios were investigated to determine the maximum compression ratio appropriate for SAR interferometry. Based on the scenes studied in this paper, it was concluded that a reduction from 8 to 4-bits/sample was the maximum data reduction ratio appropriate for precision SAR interferometry, while 8 to 3-bits/sample and 8 to 2-bits/sample encoding were only appropriate for lessdemanding wide-swath applications.
\end{abstract}

Index Terms - Block adaptive quantization, data compression, satellite interferometry.

\section{INTRODUCTION}

$\mathbf{T}$ HE EUROPEAN Space Agency's (ESA's) remotesensing satellite ENVISAT is scheduled to be launched in 1999, with the Advanced SAR (ASAR) as the SAR imaging sensor. Capable of multiple imaging modes and resolutions, ASAR will offer users a flexible all-weather, day or night, high-resolution imaging sensor. However, the quantities of SAR data that can be produced is constrained by the size of onboard data storage and downlink bandwidth [1].

To address this constraint, while supporting the wide-swath applications demanded by users, an onboard data reduction scheme was proposed. The data reduction algorithm was required to be relatively simple at the encoder to provide high reliability and throughput while using minimal satellite resources. In addition, it was deemed desirable to offer multiple data reduction ratios, as the various operating modes have different image quality/data volume requirements.

To meet the onboard data reduction criteria of the ENVISAT ASAR, a SAR signal-data reduction algorithm called Flexible Block Adaptive Quantization (FBAQ) was developed by MacDonald Dettwiler and Associates (MDA), Vancouver, B.C.,

Manuscript received December 27, 1995; revised July 7, 1997. This work was performed under ESA Contract 10737/94/NL/JG.WO 03, with Saab-Ericsson Space as the prime contractor and MacDonald Dettwiler as the subcontractor. The technical work was performed in the MDA/NSERC Industrial Research Chair at the University of British Columbia. This work was supported by MDA and NSERC.

I. H. McLeod was with the Department of Electrical Engineering, University of British Columbia, Vancouver, B.C., Canada V6T 1Z4. He is now with MacDonald Dettwiler and Associates, Richmond, B.C., Canada V6V 2J3 (email: ianc@ee.ubc.ca).

I. G. Cumming and M. S. Seymour are with the Department of Electrical Engineering, University of British Columbia, Vancouver, B.C., Canada V6T 124 .

Publisher Item Identifier S 0196-2892(98)00738-4.
Canada, for the European Space Research and Technology Center (ESTEC) in 1994 [2]. FBAQ reduces the 8-bit/sample SAR signal data to 4-, 3-, or 2-bits/sample, as selected by mission control, and a full 8-bit pass-through mode is also supported. In a previous study, several variations of the algorithm were tested and compared to other SAR data reduction techniques, such as Vector Quantization [2]-[4] (the results of a parallel study are given in [5]). Tests included both quality measures of the reduced SAR signal data and an evaluation of images produced from the signal data [6]. The version of FBAQ eventually chosen was found to deliver high image quality using an encoder suitable for implementation on a single application-specific integrated circuit (ASIC) [2] (the results of a parallel study are given in [7]). The ASIC was implemented by Saab-Ericsson Space in 1994.

However, while the quality of SAR images produced using the algorithm were evaluated with good results, it was also considered important to examine the impact of data encoding on selected applications, such as ocean wave analysis (wavemode) [8] and repeat-pass interferometry [9].

In this paper, the results of experimental studies using ERS-1 SAR data are used to assess the impact of FBAQ onboard data encoding on the accuracy of digital elevation models (DEM) produced using repeat-pass SAR Interferometry. Sections II and III describe the theory of FBAQ and interferometric SAR (InSAR), while Sections IV and V detail the experimental methodology and the results obtained during the study. Concluding remarks are given in Section VI.

\section{FBAQ}

The FBAQ SAR data reduction algorithm developed by MacDonald Dettwiler and Associates for the ENVISAT mission evolved from the Block Adaptive Quantization algorithm developed by the Jet Propulsion Laboratory (JPL), Pasadena, $\mathrm{CA}$, for the Magellan Mission to Venus [10] and later used on the Shuttle Imaging Radar Mission C (SIR-C). Whereas both JPL implementations were for a fixed data-compression ratio (8 to 2-bits for Magellan, 8 to 4-bits for SIR-C), the FBAQ algorithm allows operational selection of compression ratio from mission control. This added flexibility is important for modern SAR sensors, such as ASAR, as the different operating modes of the sensor and applications of the SAR images have different image quality and data volume requirements.

\section{A. SAR Signal Data}

To understand the rationale behind the FBAQ algorithm, we must first consider the characteristics of the raw SAR signal data to which the algorithm is applied. 
ENVISAT SAR signal data can be modeled by the following statistical properties [11]:

1) baseband complex data, sampled at 8-bits/sample (8I, 8Q);

2) zero-mean, circular Gaussian distribution of complex samples;

3) small amount of saturation in the analog to digital converter;

4) low correlation between the I and Q channels;

5) low intersample correlation in range and azimuth (the two dimensions);

6) slowly changing variance in both slant range and azimuth;

7) range and azimuth data spectrum is relatively flat.

Due to the low sample-to-sample correlation, lack of systematic patterns in the data, and relatively large bandwidth, with respect to the sampling rate, many traditional spatial-data reduction algorithms such as predictive coding or transform coding are not optimal for SAR signal data [11]. In particular, lossless data reduction algorithms generally perform poorly (yield low-compression ratios) on the relatively uncorrelated SAR signal data. However, due to the relatively low SNR levels of satellite SAR data (between 5 and $15 \mathrm{~dB}$ ), a moderate amount of added noise due to lossy-data reduction will not have a significant impact on image quality.

\section{B. Algorithm Description}

Of the SAR signal-data properties listed above, the most significant for choice of data encoding algorithm are the zero mean, circular Gaussian distribution with a slowly changing variance in both the slant range and azimuth directions. In 1960, Max [12] showed that for a nonuniform probabilitydensity function (pdf), data could be quantized more efficiently (in the mean square error sense) using nonlinear threshold and reconstruction levels that were optimized for the pdf of the data. If we assume the data to be zero mean and Gaussian distributed, the only variable that needs to be determined to calculate all threshold and reconstruction levels is the variance of the data. Note that the variance is nonstationary within the SAR data set.

To address the nonstationary nature of the data, the SAR data is first quantized to 8 bits/sample by using a standard uniform quantizer (since 8 bits is sufficient to cover the full dynamic range of the radar demodulator). FBAQ then divides the sampled data into blocks, under the assumption that the statistics within each block are stationary. Choosing the size of the block is a tradeoff: the block must be small enough that the variance of the SAR data is essentially constant within the block, but large enough that an adequate estimate of the block variance can be made.

The amount of variability within the SAR data is sensor dependent, being a function of the range chirp length and azimuth beamwidth. While it is best to choose blocks with dimensions in azimuth as well as in range, for simplicity and storage reasons, a block is taken as a number of consecutive samples from a single range line. Previous studies at MDA determined that range-direction blocks were adequate and that the algorithm was very insensitive to block sizes between 64 and 128 samples for ENVISAT data [4].

For each block of data, the FBAQ algorithm implements the following steps:

1) estimate the variance of the block of data;

2) calculate the optimum thresholds;

3) quantize the data using the optimum thresholds to less than 8 bits/sample;

4) transmit the quantized data to the ground, along with the variance estimate;

5) on the ground, calculate the optimum reconstruction levels;

6) reconstruct the quantized data.

The previous studies also concluded that different reduction ratios were appropriate for SAR data intended for different uses [4].

- 2 bits/sample: good visual quality, all image features well reproduced, no misregistration. Recommended for wideswath applications in which the image data is interpreted visually;

- 3 bits/sample: excellent image quality, radiometry, and point-target responses well preserved, no misregistration effects. Recommended for most applications with average image quality requirements.

- 4 bits/sample: best image quality, radiometry, and image statistics very close to 8-bit data levels, low levels of phase error. Recommended for precision applications involving detailed computer analysis.

For these reasons, it was decided to support four compression ratios in the ASAR scheme: 8 to 2-bit, 8 to 3-bit, 8 to 4-bit, and a pass-through mode (no encoding).

\section{SAR INTERFEROMETRY}

In satellite SAR interferometry, two images are acquired for a scene from slightly different viewing angles [13], [14]. The cross-track imaging geometry is illustrated in Fig. 1, where SAR1 and SAR2 represent the viewing positions, separated by the baseline distance $B$ at an angle $\alpha$. If the range $r$ and range difference $\delta$ are known, the off-nadir viewing angle $\theta$ can be computed from the Law of Cosines

$$
\sin (\alpha-\theta)=\frac{(r+\delta)^{2}-r^{2}-B^{2}}{2 r B}
$$

and with the satellite altitude above the datum geoid $h$, the height $z$ of point $\mathrm{P}$ is

$$
z=h-r \cos \theta \text {. }
$$

In practice, the baseline length and angle $(B, \alpha)$ must be determined accurately using tie points (points of known elevation on the ground) [13], [15], [16]. To measure $\delta$, InSAR processing utilizes the fact that a small difference in return distance, such as $\delta$, will appear as a mod $2 \pi$ phase difference $(\Phi)$ between the pixel representing point $\mathrm{P}$ in the SAR image produced by SAR1 and that produced by SAR2. The phase differences between the two images are measured by registering the images to a subpixel level, then multiplying one image by the complex conjugate of the other to form an 


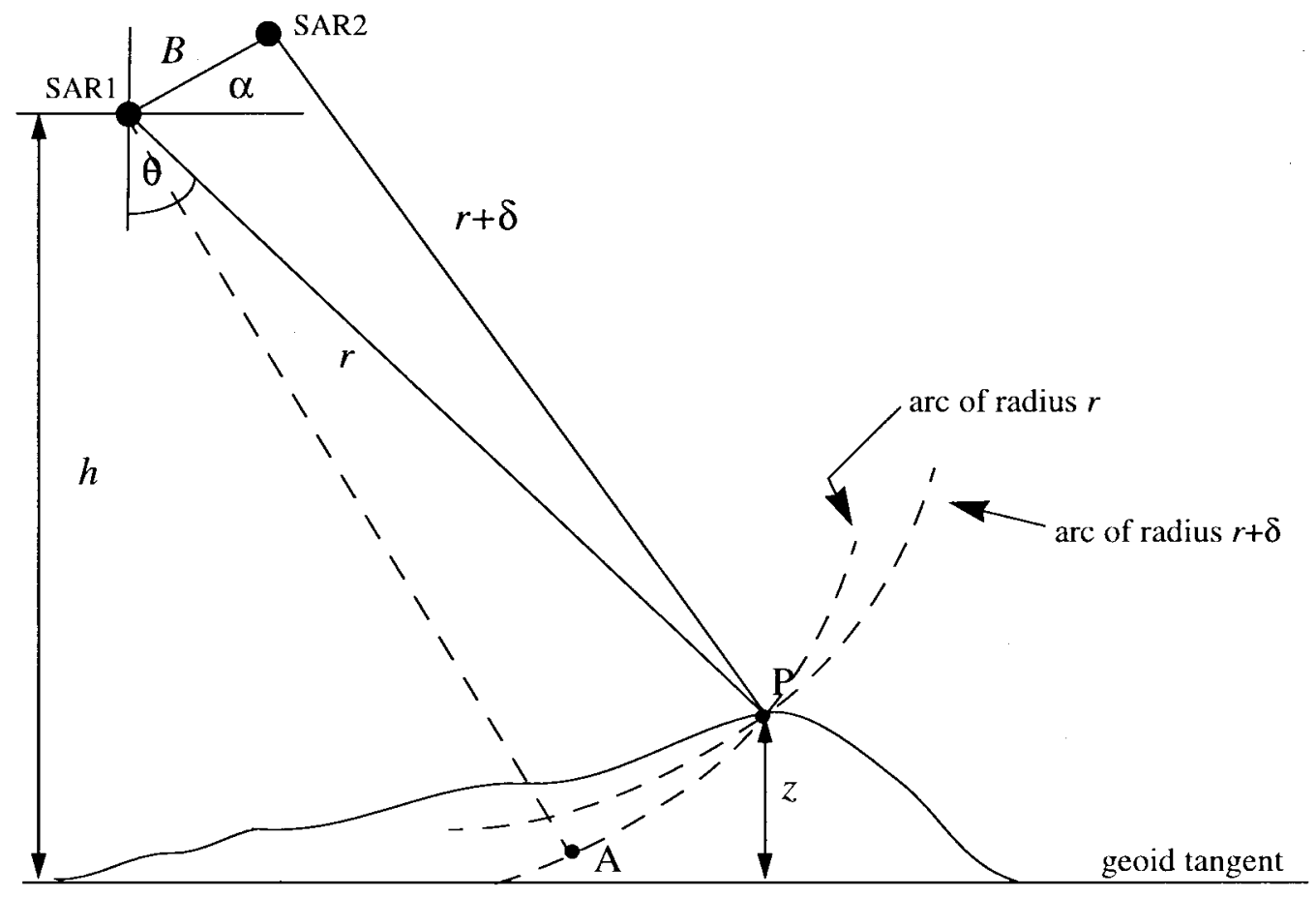

Fig. 1. Satellite across-track InSAR geometry.

interferogram. The interferogram phase is thus a map of $\Phi$ at each pixel location in the registered images. For satellite InSAR, the relation at a given pixel can be expressed as

$$
\Phi=\phi_{2}-\phi_{1}=\left[\left(\frac{4 \pi}{\lambda}\right) \delta+\phi_{\text {noise }}\right]_{\bmod 2 \pi}
$$

where $\phi_{1}$ and $\phi_{2}$ are the phase of the complex pixel representation point $\mathrm{P}$ in the SAR image produced by SAR1 and SAR2, respectively. The term $\phi_{\text {noise }}$ accounts for any phase noise present on the interferogram that make the measurement of the phase difference more difficult. There are many sources of such phase noise [16], [17], but for this study we are most concerned with the increase in $\phi_{\text {noise }}$ caused by the FBAQ algorithm.

The relation between phase noise $d \Phi$ and height-estimation uncertainty $d z$ in the DEM can be illustrated by differentiating (1)-(3), with respect to $\Phi$

$$
\begin{aligned}
\frac{d z}{d \Phi} & =r \sin \Phi\left(\frac{d \theta}{d \Phi}\right) \\
& =\frac{r \sin \theta}{\cos (\alpha-\theta)}\left(\frac{\lambda}{4 \pi B}+\frac{\lambda^{2} \Phi}{r B(4 \pi)^{2}}\right) .
\end{aligned}
$$

The inclusion of $r$ (on the order of $850 \mathrm{~km}$ ) in the denominator of the second term within the brackets of this equation makes it negligible compared to the first term, and it may be removed from the expression. From the InSAR geometry, the baseline component normal to the look direction $\theta$ can be called $B_{n}$, and we can use

$$
B_{n}=B \cos (\alpha-\theta)
$$

Assuming a small range of look angles, as is the case for scenes processed in this study, the simplified (4) can now be rewritten as

$$
d z=\left(\frac{r \lambda \sin \theta}{4 \pi B_{n}}\right) d \Phi
$$

Thus, if the rms level of phase error added to an interferogram, due to data reduction techniques, can be measured $(d \Phi)$, the resulting rms error in height estimates $(d z)$ can be determined using (6).

It can be seen that for a given ERS-1 InSAR pair with phase error $d \Phi$, the dominate factor in determining the amount of height error is the normal baseline component $B_{n}$. A larger baseline will mean less height-estimation error for a given phase error. However, the normal baseline may only be so large before the correlation between the scenes becomes too small to be useful [18] and phase unwrapping problems occur.

\section{A. Coherence Magnitude}

To measure changes in the phase-noise level of interferograms, due to FBAQ, we can simply compare interferograms produced by using data that has been FBAQ encoded/decoded to the original interferogram. However, it is important to consider the noise added by FBAQ within the context of the noise already present from other sources. To do this, we need an estimate of the phase-noise levels of the original (unencoded) interferograms. This can be accomplished through measurement of coherence magnitude of the data [19], [20].

Coherence magnitude provides a measure of the noise content, and hence quality, of interferograms. Assuming a constant interferogram phase over a small region, the complex coherence $(\gamma)$ between two complex images $S_{1}$ and $S_{2}$ used 


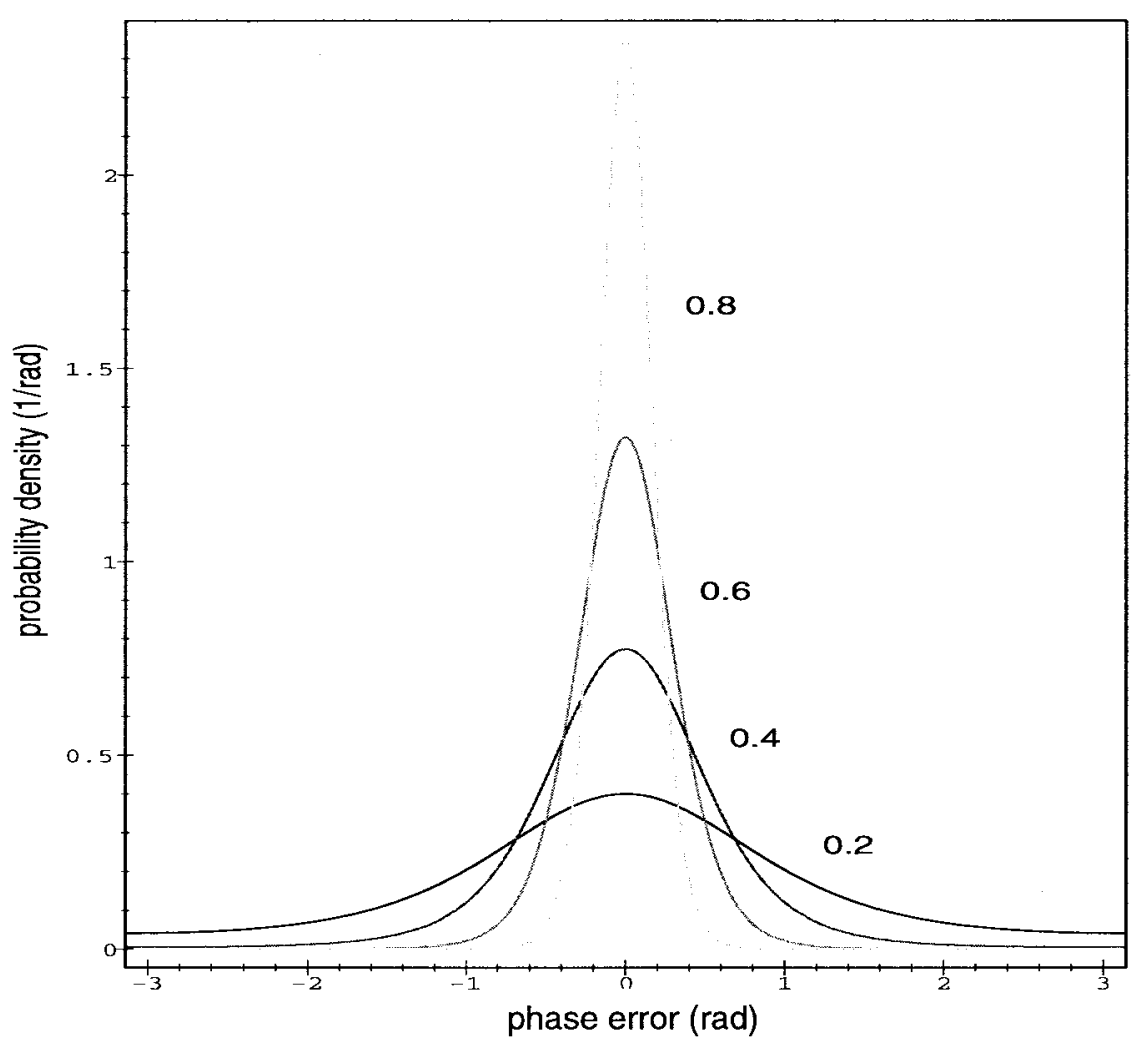

Fig. 2. Pdf of ten-look interferogram phase for coherence magnitudes $0.2,0.4,0.6$, and 0.8 .

to create the interferogram is defined as

$$
\gamma=\frac{E\left\{S_{1} S_{2}^{*}\right\}}{\sqrt{E\left\{\left|S_{1}\right|^{2}\right\} E\left\{\left|S_{2}\right|^{2}\right\}}}
$$

where $E\{\cdot\}$ represents the expected value and * represents complex conjugation.

The coherence magnitude $|\gamma|$ varies between zero (no correlation) and one (complete correlation), and the coherence phase $\left(\arg \left\{S_{1} S_{2}^{*}\right\}\right)$ is simply the phase of the interferogram after averaging. The addition of phase noise to the image data has the effect of increasing the standard deviation of $\arg \left\{S_{1} S_{2}^{*}\right\}$, which in turn lowers the value of coherence magnitude. Thus, coherence magnitude provides a means of assessing the amount of phase noise associated with an interferogram (i.e., pixels of the image with low levels of phase noise will have high coherence magnitude values, those with high levels of phase noise will have low coherence magnitude). For InSAR, this implies that areas of high coherence magnitude will be those most appropriate for processing as they have less phase noise and will thus yield better height estimates.

The relationship between coherence magnitude and standard deviation of phase error for multilooked data was derived by Lee et al. [19] and by Joughin et al. [21], assuming constant phase. The derivation by Lee shows that the phase standard deviation of an interferogram depends only upon the coherence magnitude of the data and the number of "looks." For InSAR, the number of looks means the number of interferogram pixels coherently averaged together to reduce phase noise. Joughin et al. [22] showed that the effective number of looks may be less than the theoretical number of looks for real SAR data, finding that for ERS scenes an effective number of looks between eight and 12 was appropriate for a two range by ten azimuth sample averaging window. The probability distributions are of the same form, but the effective number of looks should be substituted for the theoretical value.

Fig. 2 shows the pdf's of interferogram phase differences that were derived by Lee for ten-look smoothing and various levels of coherence magnitude. The standard deviation of these distributions provides an estimate of phase-noise level. It is clear that, as coherence magnitude increases, the standard deviation of the phase distribution becomes smaller. Note that, for a coherence magnitude of zero, the phase distribution would be uniform. As coherence magnitude increases, the phase distribution narrows in a Gaussian-like manner, until at a coherence magnitude level of one, a delta distribution occurs (zero phase noise).

Fig. 3 shows phase standard deviation versus coherence magnitude for one-look, ten-look, and 20-look smoothing. These curves provide a way to use coherence magnitude to estimate phase noise. For example, from the curves we see that a region of the interferogram with a coherence magnitude of 0.8 will have a phase uncertainty of about $52^{\circ} \mathrm{rms}$ for the single-look image, but only about $10^{\circ} \mathrm{rms}$ in the ten-look case.

The change in coherence magnitude, due to the addition of a given amount of phase noise, depends upon the initial level of coherence magnitude. For the multilook case, a small amount of phase noise added to an area of high coherence (such as 


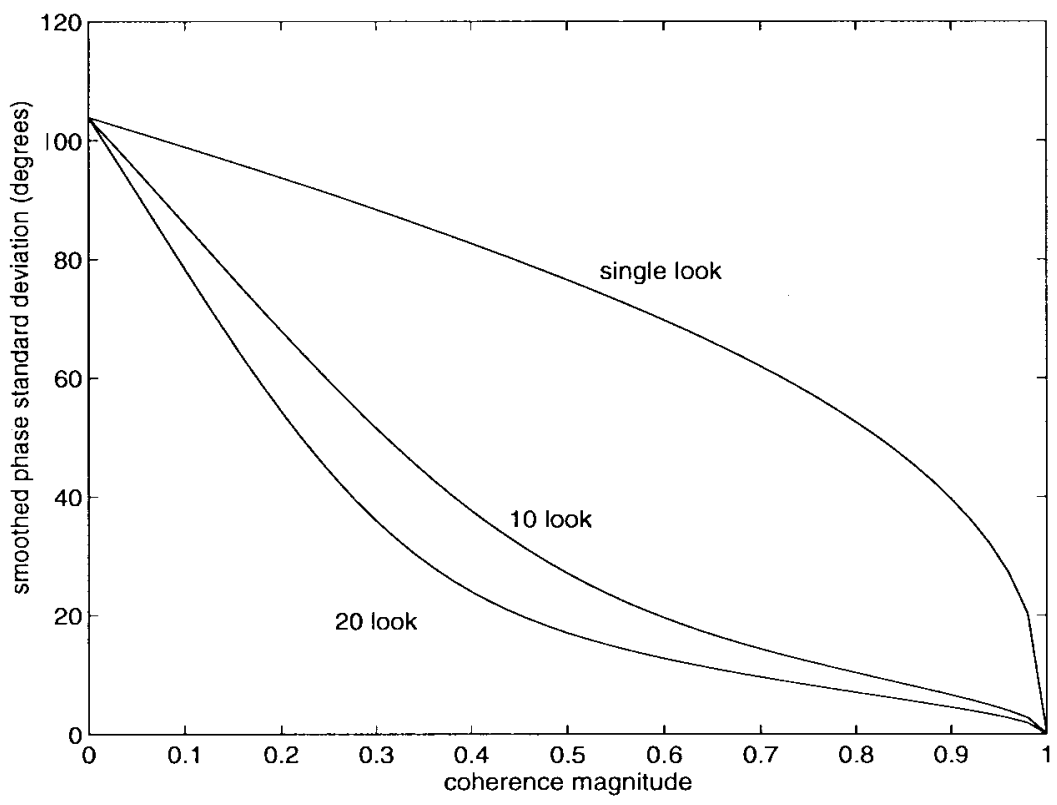

Fig. 3. Interferogram phase standard deviation versus coherence magnitude for one, ten, and 20 looks.

TABLE I

INSAR PRocessing PARAMETERS

\begin{tabular}{l|c|c}
\hline \multicolumn{1}{c|}{ Parameter (symbol) } & Sardinia & Toolik Lake \\
\hline \hline wavelength $(\lambda)$ & $0.0566 \mathrm{~m}$ & $0.0566 \mathrm{~m}$ \\
\hline look angle $\left(\theta_{\mathrm{c}}\right)$ at center swath & 22.4 degrees & 24.0 degrees \\
\hline slant range sampling interval $(\Delta \mathrm{r})$ & $7.905 \mathrm{~m}$ & $7.905 \mathrm{~m}$ \\
\hline baseline normal to slant range $\left(\mathrm{B}_{\mathrm{n}}\right)$ & $126 \mathrm{~m}$ & $40.4 \mathrm{~m}$ \\
\hline baseline parallel to slant range $\left(\mathrm{B}_{\mathrm{p}}\right)$ & $65 \mathrm{~m}$ & $98.2 \mathrm{~m}$ \\
\hline $\begin{array}{l}\text { slant range distance to center range } \\
\text { pixel }\left(\mathrm{r}_{\mathrm{c}}\right)\end{array}$ & $844 \mathrm{~km}$ & $858 \mathrm{~km}$ \\
\hline
\end{tabular}

0.8) will cause a relatively large drop in coherence magnitude, while the same amount of phase noise added to an area of low coherence will cause a much lower change in the coherence magnitude level. For example, much more phase noise would have to be added to cause the coherence magnitude to move from 0.3 to 0.2 than from 0.8 to 0.7 in the multilook case.

\section{EXPERIMENTAL METHODOLOGY}

In order to examine the effect of data encoding on ENVISAT, it was best to use available data with properties similar to those of the future system. These properties are dependent upon system parameters, such as imaging geometry, transmitter power, wavelength, polarization, and level of signal quantization. As data with properties very close to those expected for ENVISAT can be obtained from data from the current ESA SAR satellite ERS-1 [4], it was selected for use in this study.

\section{A. Scene Selection}

Two InSAR scenes with quite different characteristics were selected for the study. The first is Sardinia, Italy, consisting of ERS-1 data acquisitions taken six days apart during orbits
241 (Aug. 2, 1991) and 327 (Aug. 8, 1991). The second pair is Toolik Lake, Alaska, from orbits 943 (Sept. 20, 1991) and 1029 (Sept. 26, 1991). The InSAR processing parameters used for the two scenes are given in Table I.

\section{B. Data Preconditioning}

In order to model 8-bit, zero-mean ENVISAT data, the 5-bit, 15.5-mean ERS-1 data was preconditioned to 8-bit zeromean format. This essentially involves subtracting the mean, adding a small amount of noise to the data set, reducing the saturation levels of the histogram to 8-bit levels using exponential noise, and requantizing the data to 8-bit format. The addition of noise to the data will reduce the coherence magnitude slightly, but serves the important purpose of filling the data histogram and increasing the entropy of the data to the levels expected for 8-bit ENVISAT data, so that the correct statistical properties of the ENVISAT data is presented to the algorithm. This is important as the performance of FBAQ is strongly dependent on the histogram of the data. The average reduction in coherence magnitude due to the addition of the noise was measured at 0.02 , which was not substantial enough to impact the InSAR processing. 


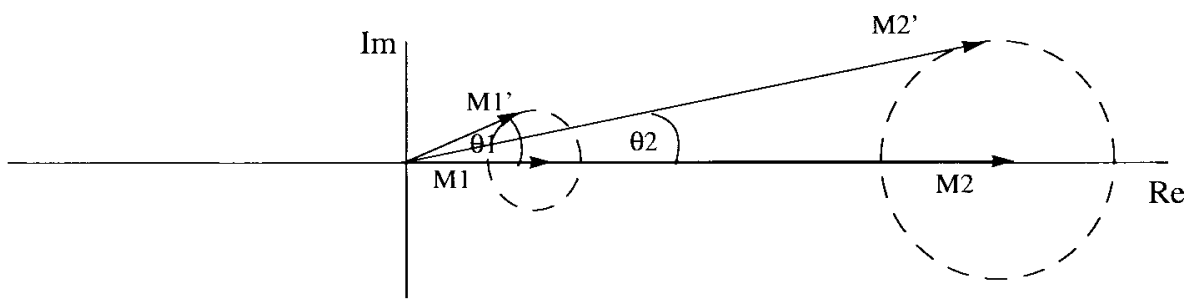

Fig. 4. Relation of FBAQ encoding phase error to magnitude. M1 represents a low-magnitude (dark) SAR image sample. M2 represents high-magnitude (bright) image sample. Note $\theta 1>\theta 2$, despite the fact that the error level for M2 has been exaggerated.

In order to allow useful interpretation of the results despite the increased noise levels, the following method was used.

- The created 8-bit scene was treated as the "original" ENVISAT data, and all error measurements due to encoding were taken relative to this scene.

- The coherence magnitude levels of each pixel in the smoothed interferogram created from the 8-bit data were measured and called the "original" coherence magnitude.

- The changes due to the encoding process were measured for each pixel in the smoothed interferograms created from the data that had been compressed using 4-, 3-, and 2-bit FBAQ. For each pixel, the change experienced due to encoding was related to the "original" coherence magnitude of the pixel.

Thus, the effect of the encoding on the interferograms is quantified as a function of level of coherence magnitude prior to encoding. The only impact of the additional noise is to slightly change the distribution of the coherence magnitude values contained in the interferogram. Both high and low coherence regions still existed and the impact of FBAQ encoding on these coherence levels was measured.

\section{Interferogram Processing Steps}

After preconditioning the ERS-1 SAR data to ASAR format, the data was encoded/decoded using FBAQ. The following processing steps were then applied to both the original (unencoded) data and the FBAQ encoded/decoded data:

1) process SAR signal data into single look complex (SLC) images;

2) register the images to the subpixel level;

3) oversample the images by a factor of two, and form an interferogram;

4) remove flat earth fringes in range and any azimuth phase ramps;

5) smooth the images using coherent averaging;

6) measure interferogram phase error and coherence magnitude;

7) determine incremental DEM height-estimation error due to FBAQ using (6).

\section{Registration and Phase Unwrapping}

In the above methodology, it was important to ensure that the same processing parameters were used at each step in the processing chain to isolate the errors due to FBAQ. Thus, only the original images were used to determine the registration shifts, which were then applied to all the other encoded images.
Phase unwrapping is the process of removing the mod $2 \pi$ wraparound of the interferogram phase. It is needed if absolute height measurements are to be obtained, though it is not needed in this study, as DEM error levels can be estimated through the use of (6). Phase unwrapping was not attempted in this study due to the difficulties in ensuring consistent processing across scenes, since phase unwrapping often requires a certain level of manual intervention for satellite SAR data.

However, one important concern is that FBAQ might make the registration or phase unwrapping processes more difficult. To address this issue, a separate experiment was performed for both registration and phase unwrapping. For registration, the required shifts in azimuth and range were also estimated using only FBAQ encoded/decoded images, and the results compared to the shifts determined for the original. For phase unwrapping, the number and location of phase residues were monitored to determine if FBAQ was causing a significant change to the phase residue levels within the interferogram. Phase residues are discontinuities in the phase of the interferogram and represent a limiting condition of phase unwrapping algorithm effectiveness [23]. A substantial increase in the number of phase residues would indicate that the difficulty of the phase unwrapping process had been increased by the FBAQ encoding.

\section{EXPERIMENTAL RESULTS}

\section{A. Image Phase Noise}

To understand the impact of phase noise on the interferogram, we must first consider how it is added to the images that form the interferogram. FBAQ quantization noise is added to the real and imaginary components of the 8-bit SAR signal data separately. Larger levels of noise are added to the higher sample values since they are quantized more coarsely. As shown in Fig. 4, when the complex noise (represented by the dashed circle in the complex plane) is added to two possible magnitude vectors $\mathrm{M} 1$ and $\mathrm{M} 2$, the resulting phase noise of $\mathrm{M}^{\prime}$ and $\mathrm{M} 2^{\prime}$ are not equal. Specifically, the phase noise added to the bright areas of the image (those with a large magnitude, such as M2) is less than that added to dark samples (represented by the short M1 vector). As Fig. 4 illustrates, this can be true even if higher levels of noise are added to the bright samples, as shown by the larger radius of the complex noise added to M2. After image processing, this effect is also noticeable in the bright and dark regions of the SAR image.

The effect described in Fig. 4 was observed in both the Sardinia and Toolik Lake scenes. Fig. 5 illustrates this inverse 

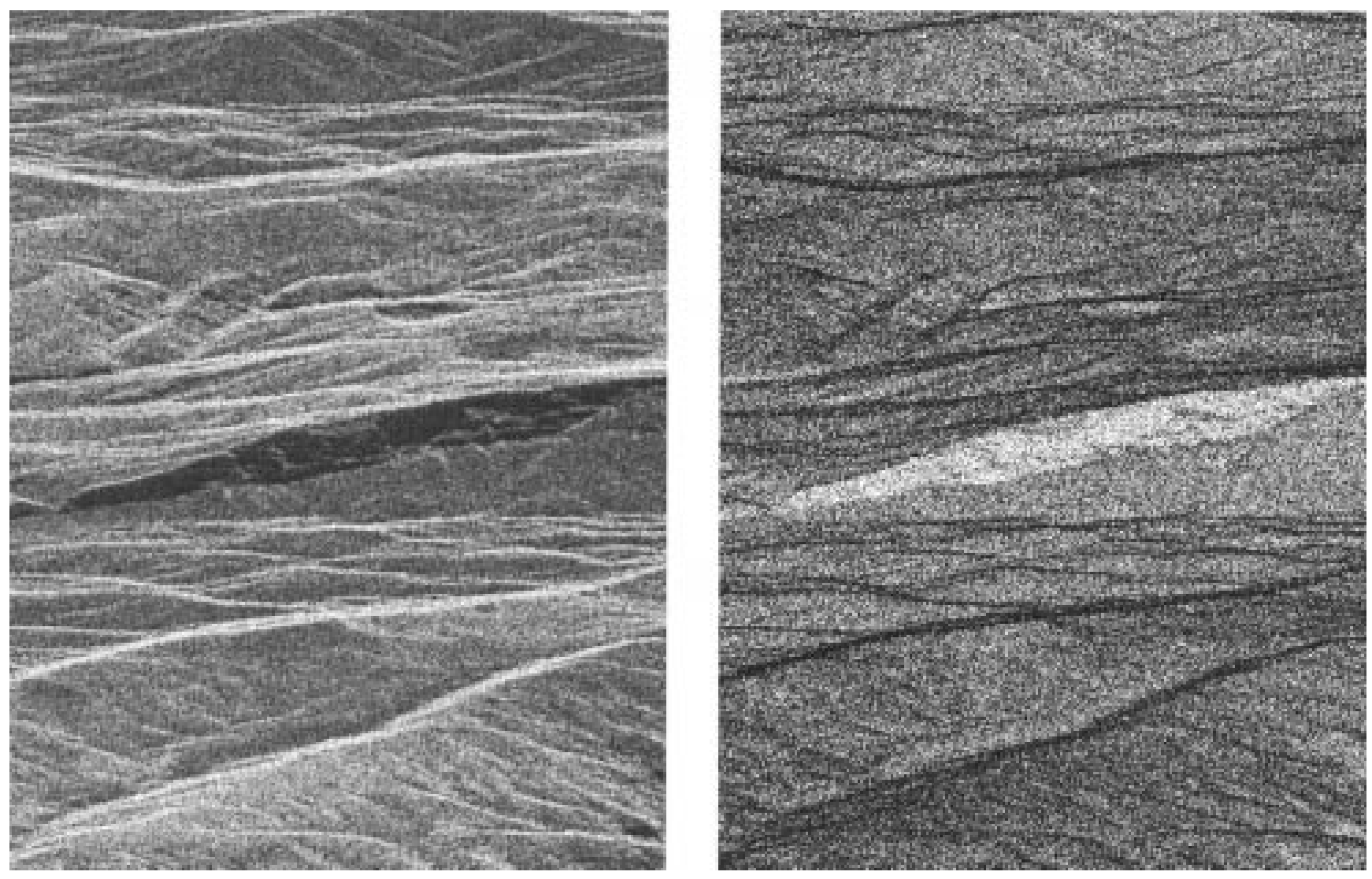

(a)

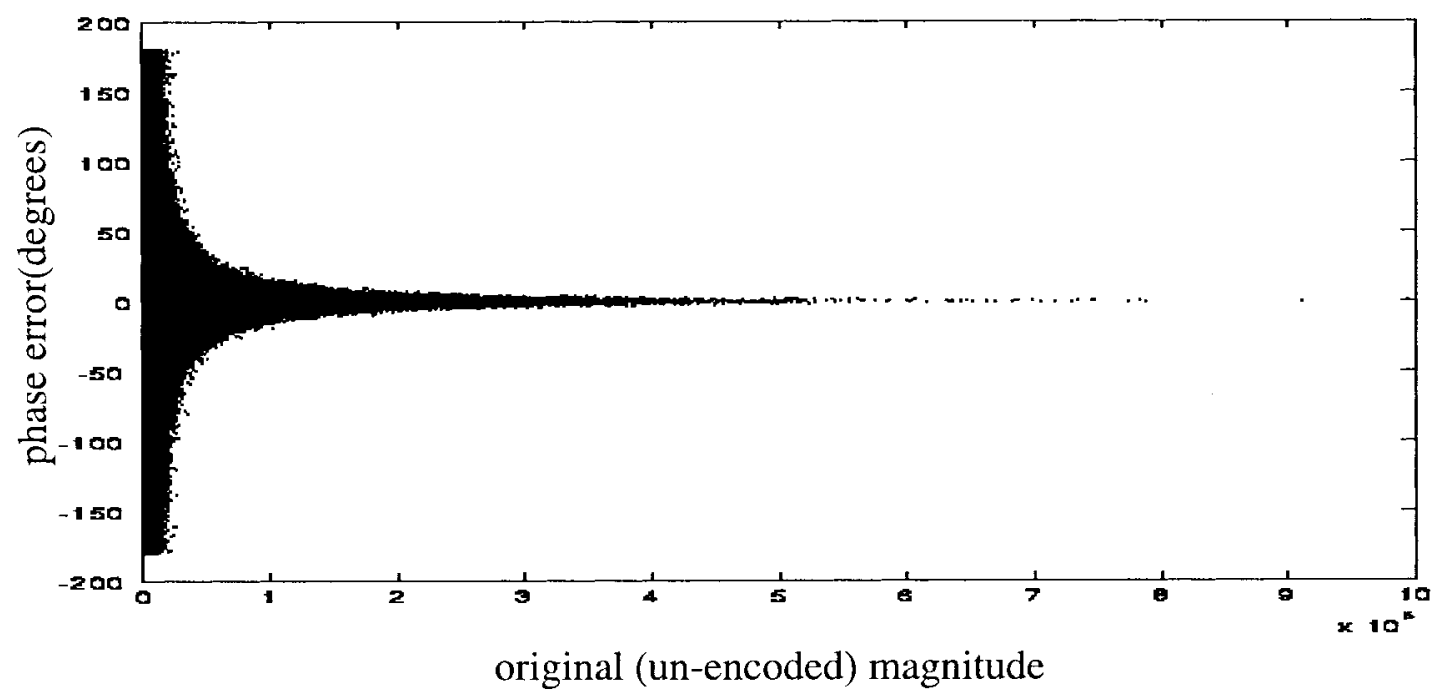

(b)

Fig. 5. SLC image of Sardinia and corresponding phase noise due to 3-bit FBAQ encoding. Phase noise has been multiplied by ten to show detail, i.e., all phase errors greater than 25.5 appear at maximum brightness (orbit 241, slant range vertical, azimuth horizontal). Scatter plot shows image magnitude versus FBAQ phase noise for 3-bit encoding.

relationship between image brightness and FBAQ phase noise using real SAR data from the study. The magnitude and 3bits/sample FBAQ phase error of the SLC Sardinia scene are shown. Note how the dark area in the center of the scene experiences the most FBAQ phase noise. The scatter plot in Fig. 5 shows clearly the trend toward higher levels of phase error in the darker (lower magnitude) areas.
This trend has important implications on interferometric processing. Bright pixels correspond to areas of high radar return in the image and, hence, areas of high SNR. A strong relationship exists between the SNR level of the images and the coherence magnitude of the interferogram. Assuming that temporal and baseline decorrelation (phase noise added due to changes on the ground between image acquisitions and due to 
TABLE II

Image and Raw Interferogram Global FBAQ Phase-Noise Measurements

\begin{tabular}{l|c|c|c}
\hline & $\begin{array}{c}\text { Image 1 rms FBAQ } \\
\text { phase noise } \\
\text { (degrees) }\end{array}$ & $\begin{array}{c}\text { Image 2.FBAQ } \\
\text { rms phase noise } \\
\text { (degrees) }\end{array}$ & $\begin{array}{c}\text { Raw Interferogram FBAQ } \\
\text { rms phase noise before } \\
\text { smoothing (degrees) }\end{array}$ \\
\hline \hline Sardinia & 13.2 & 14.0 & 18.8 \\
\hline 4-bit encoding & 23.3 & 24.2 & 32.6 \\
\hline 3-bit encoding & 37.2 & 38.3 & 50.7 \\
\hline 2-bit encoding & & & \\
\hline \hline Toolik Lake & 10.9 & 10.9 & 15.3 \\
\hline 4-bit encoding & 18.7 & 18.7 & 26.1 \\
\hline 3-bit encoding & 30.8 & 30.7 & 42.2 \\
\hline 2-bit encoding & & & \\
\hline
\end{tabular}

differences in ground reflectivity when viewed from different angles) are not dominant, the primary source of decorrelation in the interferogram will be receiver noise, which is directly related to the SNR of the original images used to form the interferogram. Thus, areas of low SNR in the images will produce areas of low SNR within the interferogram, which in turn will have a low value of coherence magnitude. The relationship between SNR and coherence magnitude can be expressed as

$$
|\gamma|=\frac{1}{\sqrt{\left(1+\mathrm{SNR}_{1}^{-1}\right)\left(1+\mathrm{SNR}_{2}^{-1}\right)}}
$$

where $\mathrm{SNR}_{1}$ and $\mathrm{SNR}_{2}$ are the $\mathrm{SNR}$ values for the images used to form the interferogram [20]. Since areas of high coherence are those suitable for InSAR processing, it is an important observation that little phase noise is added in those regions due to FBAQ.

Finally, it was noted that the FBAQ phase error added to the image met the following criteria:

a) was zero mean;

b) was uncorrelated with the original phase of the image samples;

c) had a histogram that was approximately Gaussianshaped.

The FBAQ phase error in the two images used to form the interferogram was assumed to be statistically independent. This assumption is reasonable given that the interferogram phase error was nearly equal to the quadratic sum of the phase error of the two images, as shown in Table II. However, it is the local phase error statistics, specifically those in the high coherence magnitude regions, which are most important for InSAR DEM generation.

\section{B. Interferogram Analysis}

In Fig. 6, the smoothed magnitude and phase of the interferograms used in this study are shown. The scenes are approximately $18 \mathrm{~km}$ in range by $3 \mathrm{~km}$ in azimuth and have been smoothed using two range samples by ten azimuth samples to produce square ground pixels. One phase change of black to white is one rotation from $-\pi$ to $\pi$, or one phase fringe. These scenes were chosen for their differing statistical properties, which represent extremes in InSAR processing. The rugged Sardinia scene contains widely varying levels of SNR and coherence magnitude and a high density of phase fringes. The Toolik Lake scene has relatively high SNR across the whole scene and high coherence magnitude. The combination of a short baseline and relatively flat terrain means that the phase varies very slowly across the interferogram after the removal of flat-earth fringes and only two phase fringes are visible.

When FBAQ encoding is applied, the result is an increase in the phase-noise levels of the smoothed interferograms. Fig. 7 shows the histograms of FBAQ phase noise for the Toolik Lake scene at various levels of data reduction. In all cases, the phase noise is zero-mean, approximately Gaussian shaped, with decreasing standard deviation for decreasing reduction ratio. Similar results were obtained for the Sardinia scene. Note that the global rms value of the phase noise has been reduced considerably by the smoothing operation, as seen by the decreased rms value of the distributions in Fig. $7 .^{1}$

However, the relative impact on adding phase noise to the interferogram can only be calculated if the local level of phase noise prior to encoding is considered. As previously mentioned, this initial phase noise is directly related to the coherence magnitude of the original interferogram. This is illustrated in Fig. 8, where the amount of phase noise added to each pixel is shown as a function of coherence magnitude prior to data encoding. The fact that the Sardinia and Toolik Lake results are very similar, despite the large differences in their interferograms, shows the importance of considering the phase noise added to different pixels of the interferogram, in the context of their coherence magnitude prior to data encoding. Although the scenes are extremely different, there is a very strong correlation between the original coherence magnitude level of the interferogram pixels and the level of phase noise added by FBAQ. Specifically, it was found that

\footnotetext{
${ }^{1}$ Compare the Fig. 7 rms values with the values given in Table II for the unsmoothed case.
} 

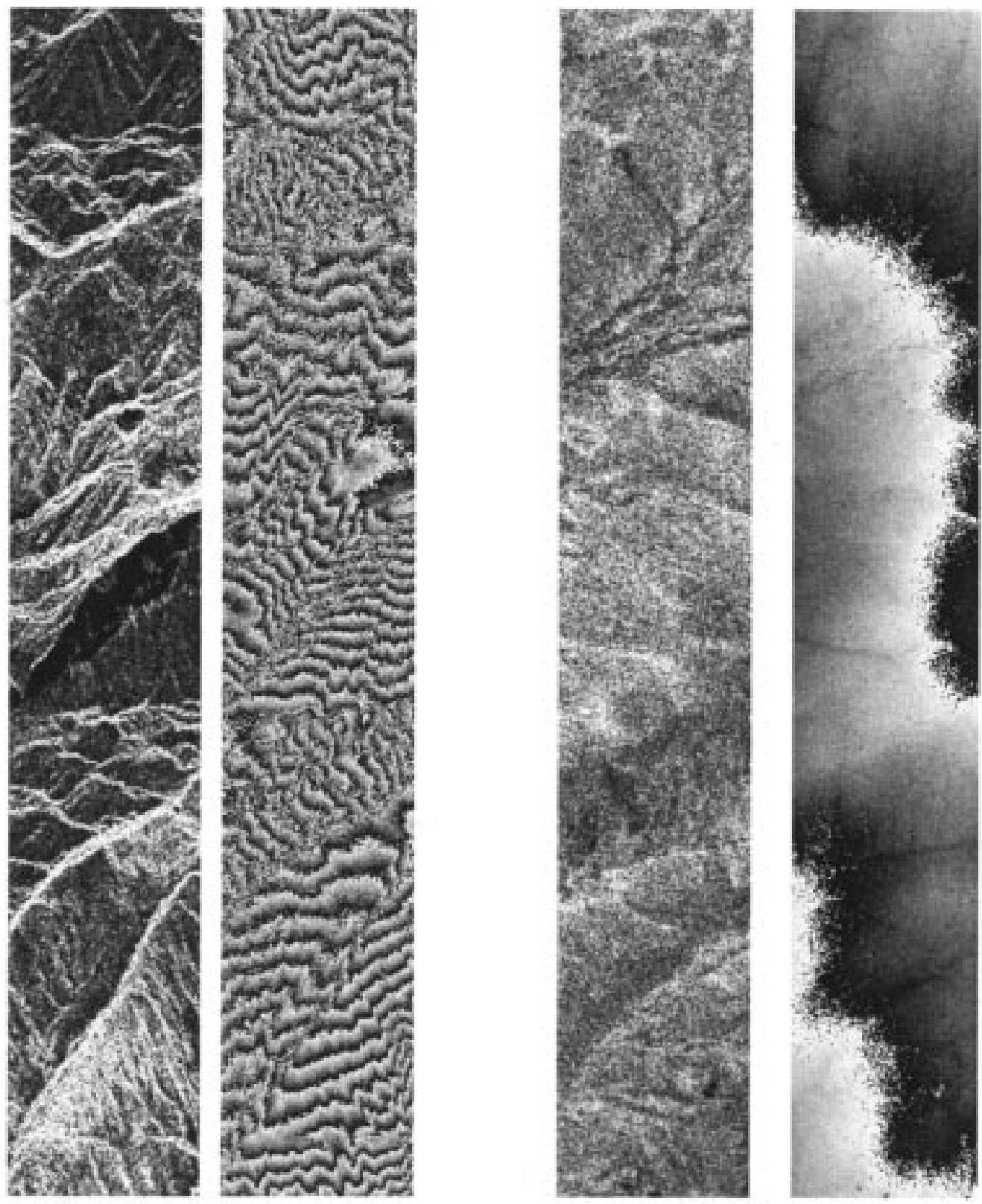

Fig. 6. Smoothed original image magnitude and interferogram phase for Sardinia and Toolik Lake scenes used in this study.

less FBAQ phase noise was added to the regions of high coherence magnitude, as explained in Fig. 4.

\section{Digital Elevation Model Results}

Two separate estimation procedures were used to determine the impact of the interferogram phase noise on DEM accuracy. For both, only regions with coherence magnitude levels greater than 0.35 were investigated, since these are the regions most appropriate for InSAR mapping. The first method was to simply add the variance of the FBAQ phase noise to that of the initial phase noise, as determined using coherence magnitude estimates. This is the method commonly used to estimate the impact of several independent phase-noise contributions. The total variance could then be converted to a DEM rms height uncertainty by using (6). This method assumes implicitly that both the initial interferogram phase noise and the FBAQ phase noise can be modeled as independent, zero-mean, random variables. The original DEM uncertainty is obtained from the original coherence magnitude estimates, which are related to phase uncertainty via Fig. 3 and converted to height uncertainty via (6). Since for ERS-1 the pixels are not completely uncorrelated, an effective number of looks equal to ten was assumed, based on the results of [13] and [22] for ERS-1 data.

The second method of analysis was to measure the change in coherence magnitude due to encoding, and from that determine the level of phase noise that must have been added to cause that change by using the relation between coherence magnitude and phase noise. This level of rms phase noise could then be converted to rms height error by using (6). This method has the advantage of removing the assumption of independent, zeromean, phase-noise distributions, but has practical difficulties due to the addition of bias in the coherence magnitude esti- 


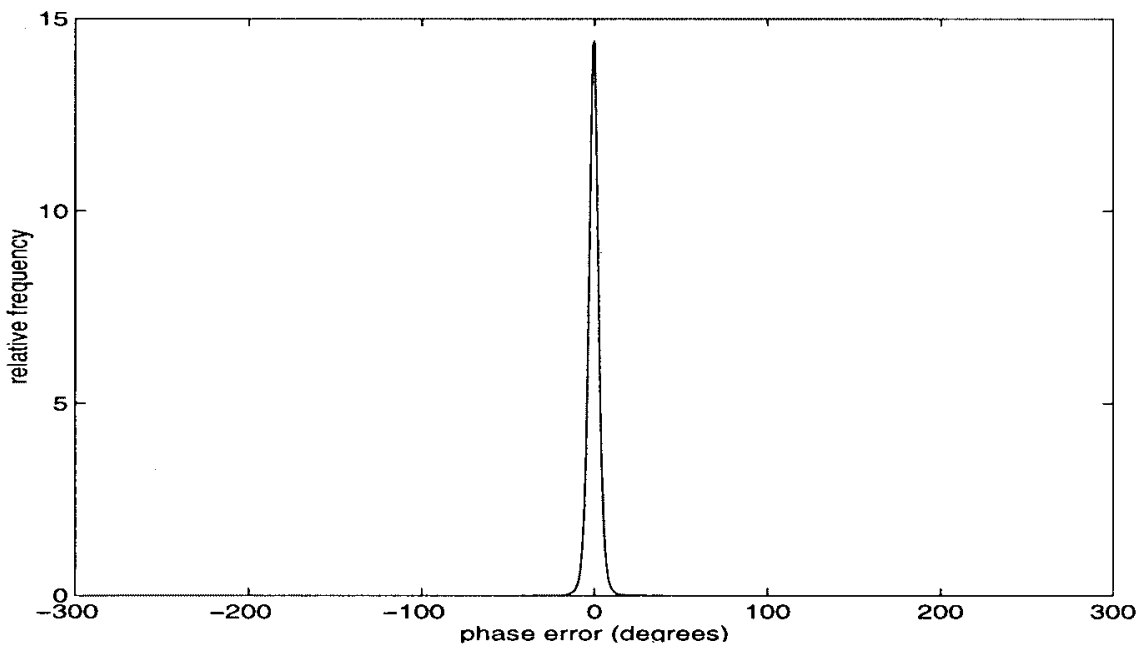

(a)

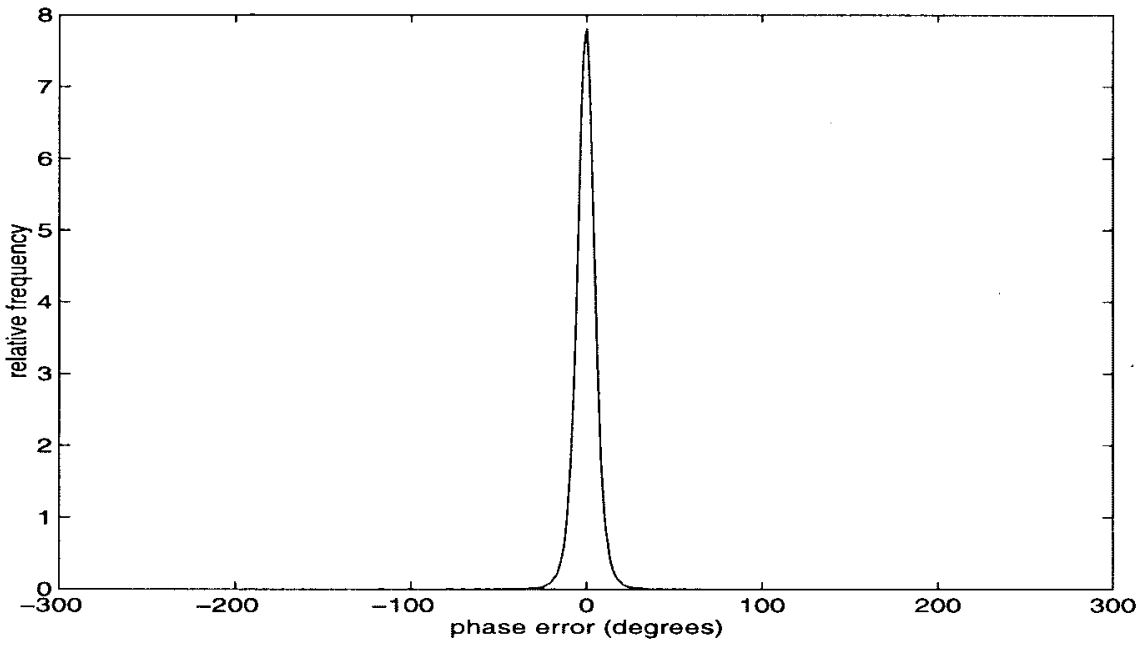

(b)

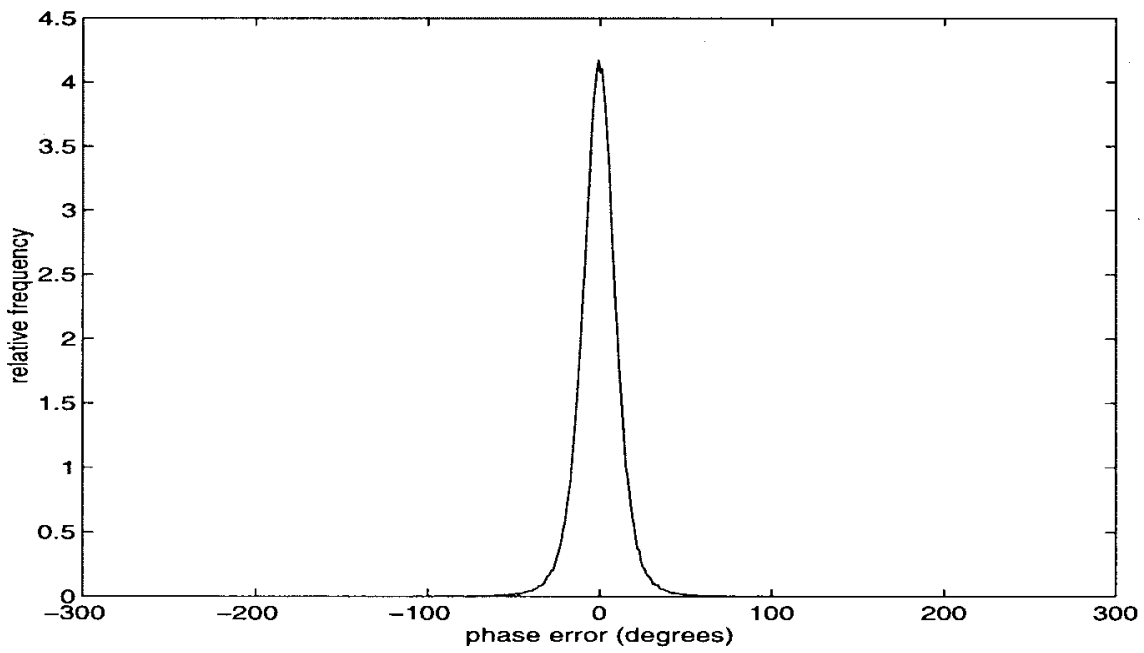

(c)

Fig. 7. Histograms of Toolik Lake smoothed interferogram phase error due to FBAQ for (a) 4-bit encoding, rms $=3.6^{\circ}$, (b) 3 -bit encoding, rms $=$ $6.8^{\circ}$, and (c) 2-bit encoding, rms $=13.0^{\circ}$. 


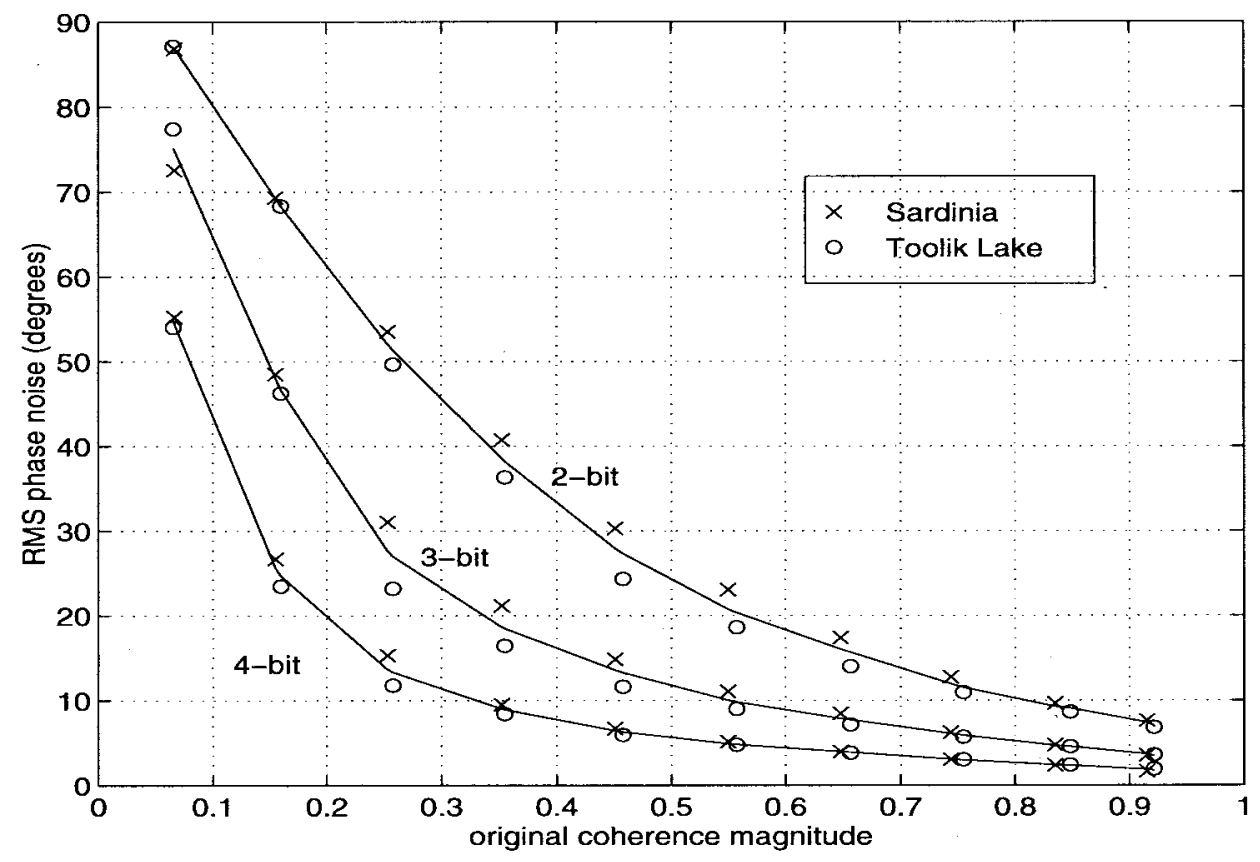

Fig. 8. Rms phase noise added to interferograms due to FBAQ encoding versus original (prior to encoding) coherence magnitude.

mator. Although bias levels are relatively small for coherence magnitude levels above 0.35 , they become significant when measuring the change in coherence magnitude due to encoding, since the small changes being measured may be on the order of the bias level. Bias is added to a greater extent at low coherence magnitude values and for a low number of looks [24]. Thus, to reduce the impact of bias, a larger smoothing window was used in the coherence magnitude calculation. Specifically, a $4 \times 20$ window was used instead of a $2 \times$ $10 .^{2}$ However, the use of a $4 \times 20$ window was only possible for the Toolik Lake scene with its relatively constant phase. In the Sardinia scene, the phase fluctuations caused by terrain changes make the use of a $4 \times 20$ window impractical without implementing a phase compensation algorithm, which was not done for this study. The $4 \times 20$ results were then interpolated to the same scale as the $2 \times 10$ results to make a fair comparison.

Once again, the changes due to encoding were monitored for each pixel based on the level of coherence magnitude of the pixel prior to data encoding. The rms height-uncertainty levels, as a function of initial coherence magnitude, are shown in Fig. 9 for the original, 4-, 3-, and 2-bit FBAQ encoding. As can be seen, both analysis methods produced results with very similar trends. Specifically, the 4-bits/sample encoding performance curve is very close to the original performance curve for no encoding, and the 3- and 2-bit performance curves show increasing levels of rms height uncertainty due to data reduction. Note that the difference in the $y$-axis values between the Sardinia and Toolik Lake scenes is due to the difference in perpendicular baseline length (126 versus 40.4 $\mathrm{m})$. To account for this effect, error measures in this study were

\footnotetext{
${ }^{2}$ Note that although bias is a problem when measuring the change in coherence magnitude, its impact on the rest of the study is extremely limited, as we focus our analysis on relatively high levels of coherence magnitude, where the effect of bias on the coherence magnitude estimator is minor.
}

taken on a percentage basis, which removes the baseline-length dependency of the results.

The estimate obtained through addition of variances yielded the higher error measure, which was adopted as an upper bound on the error. For the estimate obtained through addition of the phase-noise variances, the percentage increase in rms height uncertainty for each coherence magnitude level was found. The values for the 4-bit encoding varied between 2.3 to $4.2 \%$ for the Sardinia scene and between 1.8 and $5.0 \%$ percent for the Toolik Lake scene. Based on these results, the average percentage increase was calculated to be $2.9 \%$ for the Toolik Lake scene and $2.3 \%$ for the Sardinia scene, and thus, both were less than $3 \%$. An average rms increase in height uncertainty of about $1.5 \%$ was observed for the estimate obtained through measuring changes in coherence magnitude. It is important to note that the $3 \%$ increase can be viewed as a worst-case estimate, since it assumes perfect knowledge of the InSAR geometry. In actual DEM generation, a finite error term is introduced, due to uncertainties in determining the satellite positions and, hence, the baseline length and angle. The size of this error term will vary from scene to scene. As an example, if a typical error level of about 3-m rms was included in our estimates for the Sardinia scene, the percent increase in error due to FBAQ dropped to less than $1 \%$.

The upper bound on average rms height uncertainty increases in the 3- and 2-bit FBAQ encoding cases to between about $7-10 \%$ and about $30-45 \%$, respectively. While interferometric processing was still possible for these levels of data reduction, their usefulness would be limited to DEM applications where high coverage is a priority (e.g., the TOPSAT mission [25]) as long as accuracy is not critical.

\section{Phase Unwrapping and Registration Results}

Registering the images using the FBAQ encoded/decoded images produced a negligible decrease in coherence magnitude 


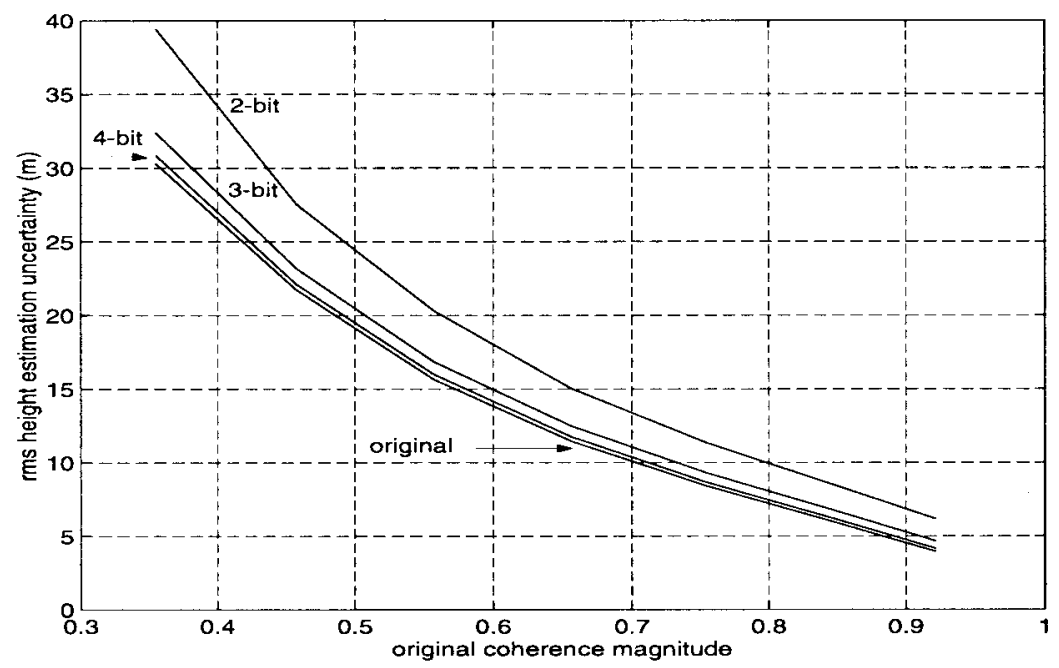

(a)

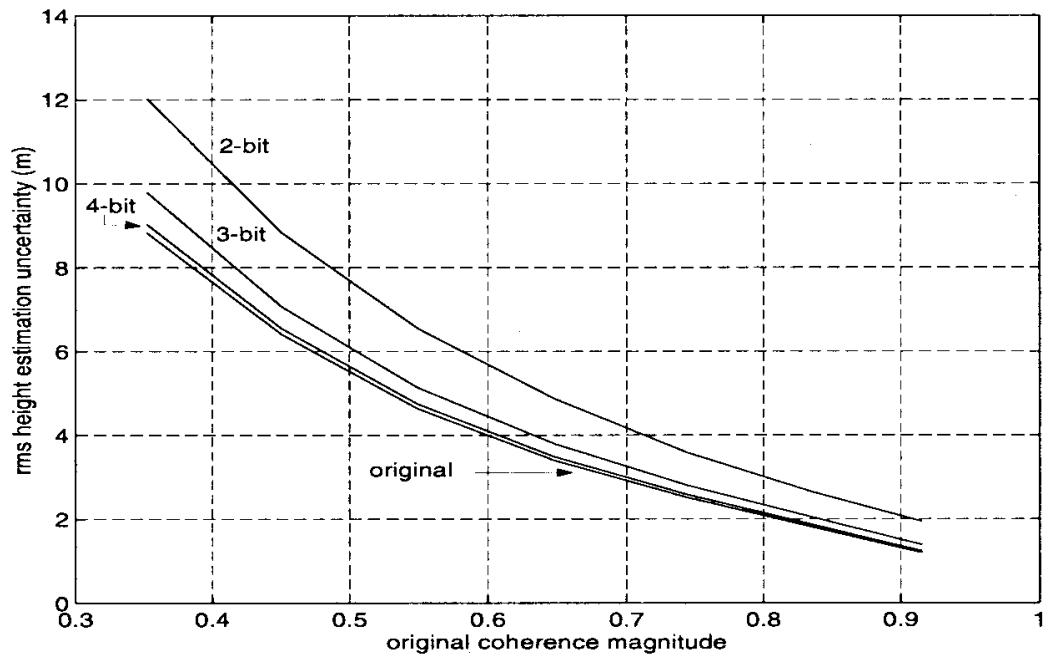

(b)

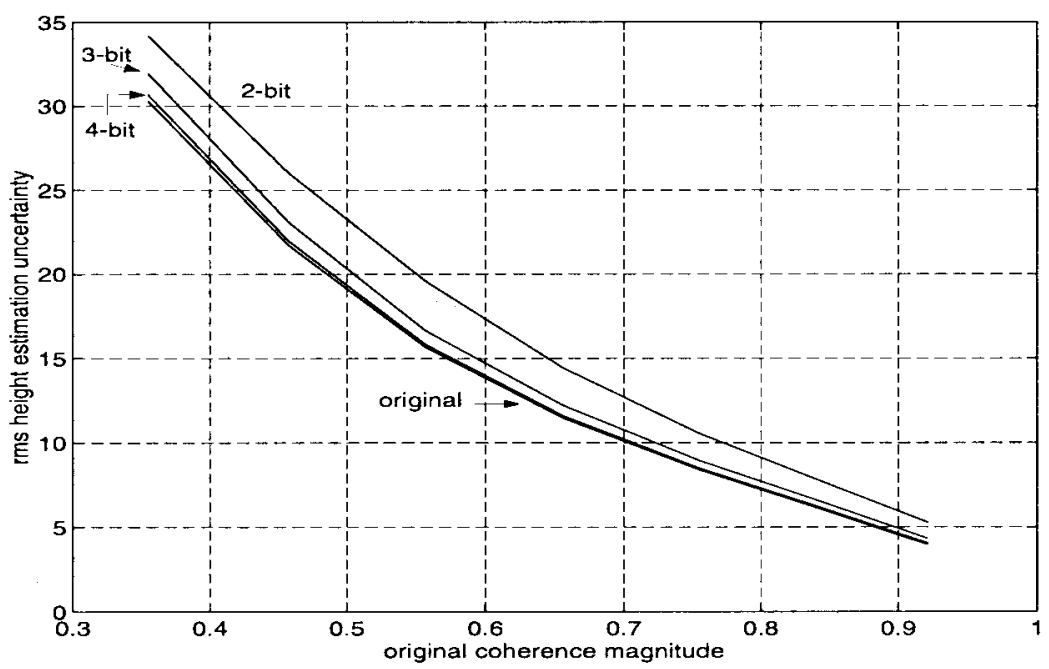

(c)

Fig. 9. Total rms height uncertainty versus original coherence magnitude for (a) Toolik Lake using estimation by addition of variances, (b) Sardinia using estimation by addition of variances, and (c) Toolik Lake using estimation by change in coherence magnitude. 
TABLE III

Phase Residue Concentrations in the SMoothed Interferogram

\begin{tabular}{c|c|c}
\hline & $\begin{array}{c}\text { Phase residue } \\
\text { concentrations for } \\
\text { Sardinia }\end{array}$ & $\begin{array}{c}\text { Phase residue } \\
\text { concentrations for } \\
\text { Toolik Lake }\end{array}$ \\
\hline \hline Original & $7.8 \%$ & $0.04 \%$ \\
\hline 4-bits/sample & $8.1 \%$ & $0.04 \%$ \\
\hline 3-bits/sample & $8.5 \%$ & $0.07 \%$ \\
\hline 2-bits/sample & $10.4 \%$ & $0.13 \%$ \\
\hline
\end{tabular}

of the resulting interferograms for all FBAQ encoding levels. It was thus possible to achieve accurate registration of the images regardless of the FBAQ data reduction level.

The phase residue concentrations of the original and FBAQ interferograms are shown in Table III, where phase residue concentration is defined as the number of phase residues divided by the number of samples in the smoothed interferogram. Note that no increase was observed for 4-bit encoding of the Toolik Lake scene. A 3\% increase was observed for the Sardinia scene. However, the Sardinia scene had many sections that were already quite noisy even for the original data, and it was observed that most of the extra phase residues were added in the regions already inappropriate for InSAR processing. Higher levels of phase residue concentrations for 3- and 2bit encoding suggest that phase unwrapping may be made a little more difficult for these encoding levels.

\section{SUMmaRY AND CONCLUSIONS}

In this study, an experiment was performed to assess the effect of FBAQ encoding on the practical application of satellite repeat pass interferometry. The results of the study can be summarized as follows.

- The impact of FBAQ on interferometric accuracy was through the addition of phase noise to the images used to form the interferogram. FBAQ was shown to add zeromean, near-Gaussian, approximately independent phase noise to the encoded images, the magnitude of which was dependent on the data reduction level chosen (more error for greater data reduction) and was inversely proportional to the local SNR of the image samples.

- Assuming a minimal amount of temporal and baseline decorrelation, the pixel brightness (SNR) of the images could be related to the coherence magnitude of the interferogram. Under this assumption, FBAQ phase error was found to be inversely related to interferogram coherence magnitude level.

- For the scenes studied, InSAR processing was possible for all FBAQ encoding levels including 2-bits/sample. Registration programs were not hampered by the FBAQ encoding noise.

- The decrease in DEM rms height accuracy due to 4bit FBAQ encoding was characterized as a function of the original, unencoded coherence magnitude of the scene. For the scenes studied, the increase in rms height uncertainty varied between 2 to $5 \%$, depending on initial coherence magnitude level, and on average was less than
$3 \%$ of the initial uncertainty of the original data. This number considers only error due to data encoding. The relative impact of the data encoding would be further reduced if other error sources were considered, such as imperfect knowledge of the InSAR geometry.

- For the scenes studied, the average decrease in accuracy for the 3- and 2-bit FBAQ was on the order of 7-12\% and $30-45 \%$, respectively, again not including calibration error. This level of error was not recommended for precision InSAR mapping, though it may be useful for large-scale applications where precision is not a priority.

- Based on analysis of the phase residues of the scenes studied, phase unwrapping should not be made more difficult due to 4-bit FBAQ encoding. The increase in the number of phase residues was minor even for the lowcoherence Sardinia scene (approximately 3\% increase) and there was no increase in phase residues for the higher coherence Toolik Lake scene. Phase unwrapping may have been more difficult in the 3- and 2-bit cases, as higher residue counts were observed for these levels. It was noted, however, that in all cases the majority of phase residues tend to be added to low coherence regions of the interferogram, which in many cases are already inappropriate for phase unwrapping.

Thus, for the scenes analyzed in this paper, 8 to 4-bit FBAQ encoding was the maximum data reduction level recommended for precision repeat-pass satellite InSAR. This level of data reduction was found to cause an average increase in rms height uncertainty of approximately $3 \%$.

\section{ACKNOWLEDGMENT}

The authors would like to thank L. Kanderhag of SaabEricsson Space (SES) and W. Wijmans of ESTEC for support and advice. Thanks also to M. Dutkiewicz, I. Burke, and G. Kuduvalli of MacDonald Dettwiler for technical advice and initial software development.

\section{REFERENCES}

[1] J. Curlander and R. McDonough, Synthetic Aperture Radar: Systems and Signal Processing. New York: Wiley, 1991.

[2] G. Kuduvalli, M. Dutkiewicz, and I. Cumming, "Synthetic aperture radar signal data compression using block adaptive quantization," in GSFC Space Earth Sci. Data Compression Conf., 1994.

[3] M. Dutkiewicz and I. Cumming, "Data encoding techniques study: SAR study," Final Rep., ESA Contract 19122/90/NL/PR(SC), Jan. 17, 1992.

[4] M. Dutkiewicz, G. Kuduvalli, and I. Cumming, "SAR pre-processing on-board study: Algorithm definition," Final Rep., ESA Contract 10135/92/NL/FM(SC), May 5, 1994.

[5] K. Strodl, U. Benz, F. Blaser, T. Eiting, and A. Moreira, "A comparison of several algorithms for on-board SAR raw data reduction," in Proc. IGARSS'94, pp. 2197-2199.

[6] M. Dutkiewicz and I. Cumming, "Evaluation of the effects of encoding on SAR data," Photogrammetric Eng. Remote Sens., vol. 60, no. 7, pp. 895-904, July 1994.

[7] R. Bertoni, L. Di Paolo, R. Di Julio, F. Impagnatiello, and F. Quaranta, "Data compression device for SAR application," in Proc. IGARSS'94, pp. 2194-2196.

[8] I. McLeod and I. Cumming, "On-board encoding of the ENVISAT wave mode data," in Proc. IGARSS'95, pp. 1681-1683.

[9] I. McLeod, I. Burke, I. Cumming, and M. Dutkiewicz, "FBAQ extended study," Final Rep., ESA Contract 10737/94/NL/JG, June 19, 1995.

[10] R. Kwok and W. Johnson, "Block adaptive quantization of Magellan SAR data," IEEE Trans. Geosci. Remote Sensing, vol. 27, pp. 375-383, July 1989. 
[11] I. Cumming, M. Dutkiewicz, and B. Akam, "Study of synthetic aperture radar data encoding techniques," in ESTEC Image Processing Workshop, June 1991.

[12] J. Max, "Quantizing for minimum distortion," IEEE Trans. Inform. Theory, vol. IT-6, pp. 7-12 and 16-21, Mar. 1960.

[13] H. Zebker, C. Werner, P. Rosen, and S. Hensley, "Accuracy of topographical maps derived from ERS-1 interferometric radar," IEEE Trans. Geosci. Remote Sensing, vol. 32, pp. 823-836, July 1994.

[14] F. Li and R. Goldstein, "Studies of multibaseline spaceborne interferometric synthetic aperture radars," IEEE Trans. Geosci. Remote Sensing, vol. 28, pp. 88-97, Jan. 1990.

[15] D. Small, C. Werner, and D. Nuesch, "Baseline modeling for ERS1 SAR interferometry," in Proc. Int. Geosci. Remote Sensing Symp., IGARSS'93, Tokyo, no. 4, pp. 1204-1206.

[16] I. Joughin, D. Winebrenner, M. Fahnestock, R. Kwok, and W. Krabill, "Measurement of ice sheet topography using satellite radar interferometry," J. Glaciol., vol. 42, no. 140, pp. 10-22, 1996.

[17] H. Zebker and J. Villasenor, "Decorrelation in interferometric radar echoes," IEEE Trans. Geosci. Remote Sensing, vol. 30, pp. 950-959, Sept. 1992

[18] C. Prati and F. Rocca, "Limits to the resolution of elevation maps from stereo SAR images," Int. J. Remote Sensing, vol. 11, no. 12, pp. 2215-2235, 1990

[19] J. S. Lee, K. Hoppel, S. Mango, and A. Miller, "Intensity and phase statistics of multilook polarimetric and interferometric SAR imagery," IEEE Trans. Geosci. Remote Sensing, vol. 32, pp. 1017-1027, Sept. 1994.

[20] D. Just and R. Bamler, "Phase statistics of interferograms with applications to synthetic aperture radar," Appl. Opt., vol. 33, no. 20, pp. 4361-4368, July 1994.

[21] I. Joughin, D. Winebrenner, and D. Percival, "Probability density functions for multilook polarimetric signatures," IEEE Trans. Geosci. Remote Sensing, vol. 32, pp. 562-574, May 1994.

[22] I. Joughin and D. Winebrenner, "Effective number of looks for a multilook interferometric phase distribution," in Proc. IGARSS'94, Pasadena, CA, pp. 2276-2278.

[23] R. Goldstein, H. Zebker, and C. Werner, "Satellite radar interferometry: Two dimensional phase unwrapping," Radio Sci., vol. 23, no. 4, pp. 713-720, 1988.

[24] J. O. Hagberg and L. M. H. Ulander, "Calibration of interferometric SAR images," EARSeL Advances Remote Sensing, vol. 4, no. 2, pp. 49-64, 1995.

[25] H. Zebker, T. Farr, R. Salazar, and T. Dixon, "Mapping the world's topography using radar interferometry: The TOPSAT mission," Proc. IEEE, vol. 82, pp. 1774-1786, Dec. 1994.

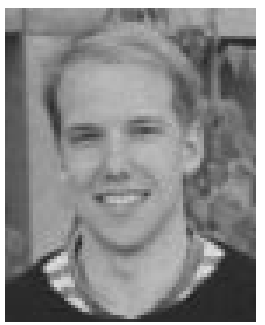

Ian H. McLeod received the B.Sc.E. degree in electrical engineering from the Queen's University, Kingston, Ont. Canada, in 1993 and the M.A.Sc. degree in electrical engineering from the University of British Columbia, Vancouver, B.C., Canada, in 1995. During studies at Queen's University, he held the Canada Scholarship in Science and Engineering, and while at UBC, studies were completed under the National Science and Engineering Research Council of Canada graduate fellowship.

He was with the Department of Electrical Engineering, University of British Columbia, until 1995. He then joined the Research Department at MacDonald Dettwiler and Associates, Richmond, B.C., Canada. Past research/development interests have included MagLev vehicle design, high-speed computer data acquisition, and solar electric vehicle design. Current activities include system engineering and algorithm development for the ENVISAT mission, along with research into hyperspectral data exploitation and data compression.

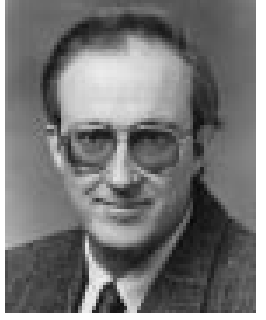

Ian G. Cumming (S'63-M'66) received the B.Sc. degree in engineering physics at the University of Toronto, Toronto, Ont., Canada, in 1961 and a Ph.D. degree in computing and automation from Imperial College, University of London, London, U.K., in 1968.

Following work in steel mill automation and sonar signal processing, he joined MacDonald Dettwiler and Associates, Richmond, B.C., Canada, in 1977. Since that time, he has developed synthetic aperture radar signal processing algorithms and worked on systems for processing polarimetric and interferometric radar data and for the compression of radar data. He has been involved in the algorithm design of the digital SAR processors delivered by MacDonald Dettwiler for SEASAT, SIR-B, ERS-1/2, J-ERS-1, and RADARSAT, as well as several airborne radar systems. In 1993, he joined the Department of Electrical and Computer Engineering at the University of British Columbia, where he holds the MacDonald Dettwiler/NSERC Industrial Research Chair in Radar Remote Sensing. The Radar Remote Sensing Laboratory supports a research staff of eight engineers and students, working in the fields of SAR processing, SAR data encoding, satellite SAR two-pass interferometry, airborne along-track interferometry, airborne polarimetric radar classification, and SAR Doppler estimation. He and his associates are Principal Investigators on the ERS Tandem Mission and on the Radarsat ADRO.

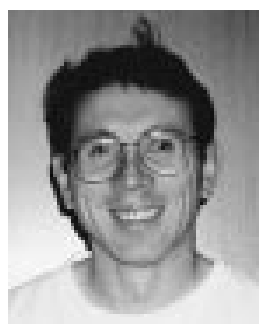

Michael S. Seymour (S'98) received the B.Sc degree from Queen's University, Kingston, Ont., Canada, in 1988 and the M.Eng. degree from McMaster University, Hamilton, Ont., Canada, in 1991. $\mathrm{He}$ is currently a Ph.D. candidate at the University of British Columbia, Vancouver, B.C., Canada.

$\mathrm{He}$ is working part-time at MacDonald Dettwiler and Associates, Richmond, B.C. His thesis topic is satellite SAR interferometry applied to map updating. 